\title{
Completeness of a Hypersequent Calculus for Some First-order Gödel Logics with Delta
}

\author{
Matthias Baaz* \\ Technische Universität Wien \\ A-1040 Vienna, Austria \\ baaz@logic.at
}

\author{
Norbert Preining ${ }^{\dagger}$ \\ Università di Siena \\ 53100 Siena, Italy \\ preining@logic.at
}

\author{
Richard Zach ${ }^{\ddagger}$ \\ University of Calgary \\ Calgary, AB T2N 1N4, Canada \\ rzach@ucalgary.ca
}

\begin{abstract}
All first-order Gödel logics $\mathbf{G}_{V}^{\triangle}$ with globalization operator $\triangle$ based on truth value sets $V \subseteq[0,1]$ where 0 and 1 lie in the perfect kernel of $V$ are axiomatized by Ciabattoni's hypersequent calculus HGIF [10].
\end{abstract}

\section{Introduction}

Gödel logics are one of the more interesting and popular many-valued logics. With the renewed interest in foundational research in fuzzy logic in the last 10-20 years, Gödel logics have come into their own. They play an important role in the algebraic study of continuous t-norm logics, but they are also interesting because of their close connection to intuitionistic logic. Takeuti and Titani [17] based their "intuitionistic fuzzy set theory" on the first-order Gödel logic with truth values from $[0,1]$, and Gödel logics have found many other applications.

Gödel logics were originally introduced by Gödel [12], and first studied in detail by Dummett [11]. He showed that propositional infinite-valued Gödel logics are axiomatized by intuitionistic logic plus the linearity axiom $(A \rightarrow$ $B) \vee(B \rightarrow A)$. More recent investigations have extended the study of Gödel logics to the first-order $[6,13,17]$ and the quantified propositional case $[4,8]$. From a proof-theoretic perspective, several versions of hyper-sequent calculi for Gödel logics have been proposed, including systems for first-order logics [3, 5, 9], and their proof-theoretic properties investigated. Ciabattoni [10] has recently extended these proof-theoretic results to include rules for the globalization, or projection, operator $\triangle$ [2].

Our aim in the present paper is to give a direct proof of completeness for the hypersequent calculus HGIF of [10]

\footnotetext{
* Research supported by FWF grant P15477-MAT

${ }^{\dagger}$ Research supported by Marie Curie Fellowship EC-MC 008054

${ }^{\ddagger}$ Research supported by the Natural Sciences and Engineering Research Council of Canada
}

which shows it to be complete not only for $\mathbf{G}_{[0,1]}^{\triangle}$, the Gödel logic with $\triangle$ on the truth-value set $[0,1]$, but for $\mathbf{G}_{V}^{\triangle}$ for any truth-value set $V \subseteq[0,1]$ the perfect kernel of which contains 0 and 1. This shows that the system HGIF applies to a broad class of truth-value sets. Moreover, we prove strong completeness, and, as a consequence, compactness. In the process we also give a direct and more elegant completeness proof of HGIF for $\mathbf{G}_{[0,1]}^{\triangle}$.

\section{Language and semantics}

We work in a usual first-order language $\mathscr{L}$ with free ( $a$, $b, \ldots)$ and bound $(x, y, \ldots)$ variables, predicate and function symbols, logical connectives $\vee, \wedge, \rightarrow$, a propositional constant $\perp$, quantifiers $\forall, \exists$, and a unary operator $\triangle$. Terms and formulas are defined in the usual way. We use $\neg$ as a defined connective; $\neg A \equiv A \rightarrow \perp$.

Definition 1 (Semantics of Gödel logic). Suppose $V$ is linearly ordered set with maximal element 1 , a minimal element 0 , and all infs and sups. An interpretation $\mathfrak{I}$ into $V$ consists of (1) a nonempty set $|\mathfrak{I}|$, the 'universe' of $\mathfrak{I}$; (2) for each $k$-ary predicate symbol $P$, a function $P^{\mathfrak{I}}:|\mathfrak{I}| \rightarrow V$; (3) for each $k$-ary function symbol $f$, a function $f^{\mathfrak{I}}:|\mathfrak{I}| \rightarrow|\mathfrak{I}|$; (4) for each free variable $a$, a value $a^{\mathfrak{I}} \in V$.

Let $\mathscr{L}^{\mathfrak{I}}$ be the language $\mathscr{L}$ extended by constant symbols for the elements of $|\mathfrak{I}|$ (so that $d^{\mathfrak{I}}=d$ ).

Given an interpretation $\mathfrak{I}$, we can naturally define a value $\mathfrak{I}(A)$ for any formula $A$ of $\mathscr{L}^{\mathfrak{I}}$. For terms $t=f\left(u_{1}, \ldots, u_{k}\right)$ we define $\mathfrak{I}(t)=f^{\mathfrak{I}}\left(\mathfrak{I}\left(u_{1}\right), \ldots, \mathfrak{I}\left(u_{k}\right)\right)$, for atomic formulas $A \equiv P\left(t_{1}, \ldots, t_{n}\right)$, we define $\mathfrak{I}(A)=P^{\Im}\left(\Im\left(t_{1}\right), \ldots, \mathfrak{I}\left(t_{n}\right)\right)$, and for composite formulas $A$ we define $\mathfrak{I}(A)$ naturally by:

$$
\begin{aligned}
\mathfrak{I}(\perp) & =0 \\
\mathfrak{I}(A \wedge B) & =\min (\mathfrak{I}(A), \mathfrak{I}(B)) \\
\mathfrak{I}(A \vee B) & =\max (\mathfrak{I}(A), \mathfrak{I}(B)) \\
\mathfrak{I}(A \rightarrow B) & = \begin{cases}1 & \text { if } \mathfrak{I}(A) \leq \mathfrak{I}(B) \\
\mathfrak{I}(B) & \text { if } \mathfrak{I}(A)>\mathfrak{I}(B)\end{cases}
\end{aligned}
$$




$$
\begin{gathered}
\mathfrak{I}(\triangle A)= \begin{cases}1 & \text { if } \mathfrak{I}(A)=1 \\
0 & \text { if } \mathfrak{I}(A)<1\end{cases} \\
\mathfrak{I}(\forall x A(x))=\inf \{\mathfrak{I}(A(u)): u \in|\mathfrak{I}|\} \\
\mathfrak{I}(\exists x A(x))=\sup \{\mathfrak{I}(A(u)): u \in|\mathfrak{I}|\}
\end{gathered}
$$

We will mostly be interested in interpretations into Gödel sets:

Definition 2. A Gödel set $V$ is a closed subset $V \subseteq[0,1]$ with $0,1 \in V$.

In the case of Gödel sets, we can interpret $\forall$ and $\exists$ as inf and sup in $\mathbb{R}$. Note that by the definition of $\neg A$, we have $\mathfrak{I}(\neg A)=0$ if $\mathfrak{I}(A)>0$ and $=1$ if $\mathfrak{I}(A)=0$.

Definition 3. For a Gödel set $V$ we define the first order Gödel logic $\mathbf{G}_{V}^{\triangle}$ as the set of all closed formulas of $\mathscr{L}$ such that $\mathfrak{I}(A)=1$ for all $\mathfrak{I}$ into $V$.

Definition 4. If $\Gamma, \Delta$ are sets of formulas (possibly infinite), we say that $\Gamma$ 1-entails $\Delta$ for $V, \Gamma \Vdash_{V} \Delta$, iff for all $\mathfrak{I}$ into $V$, whenever $\mathfrak{I}(A)=1$ for all $A \in \Gamma$, then $\mathfrak{I}(B)=1$ for at least one $B \in \Delta$.

If $V \subseteq W$ are Gödel sets, then if $\Gamma \Vdash_{W} \Delta$ also $\Gamma \Vdash_{V} \Delta$. For any $\mathfrak{I}$ into $V$ which is such that $\Im(A)=1$ for all $A \in \Gamma$ and $\mathfrak{I}(B)<1$ for all $B \in \Delta$ also is an interpretation into $W$.

This can be generalized to embeddings between truthvalue sets other than inclusion. First, note that for any map $h: V \rightarrow W$, an interpretation $\mathfrak{I}$ into $V$ induces an interpretation $\mathfrak{I}_{h}$ into $W$ by defining $\left|\mathfrak{I}_{h}\right|=|\mathfrak{I}|$ and $P^{\mathfrak{I}_{h}(\vec{u})=}$ $h\left(P^{\Im}(\vec{u})\right)$.

Definition 5. A G-embedding $h: V \rightarrow W$ is a strictly monotone mapping which preserves all existing sups and infs as well as 0 and 1 . More specifically, $h$ satisfies:

1. $h(0)=0, h(1)=1$,

2. if $a<b$, then $h(a)<h(b)(a, b \in V)$, and

3. $h(\sup \{a: a \in V\})=\sup \{h(a): a \in V\}, h(\inf \{a: a \in$ $V\})=\inf \{h(a): a \in V\}$.

Lemma 6. Suppose $h: V \rightarrow W$ is a G-embedding. (a) If $\Im$ is a $V$-interpretation, and $\Im_{h}$ is the interpretation induced by $\mathfrak{I}$ and $h$, then $\mathfrak{I}_{h}(A)=h(\mathfrak{I}(A))$. (b) If $\Gamma \Vdash_{W} \Delta$ then $\Gamma \Vdash_{V} \Delta$ (in particular $\mathbf{G}_{W}^{\triangle} \subseteq \mathbf{G}_{V}^{\triangle}$ ).

Proof. (a) By induction on complexity of formulas. (b) Suppose for some $\mathfrak{I}$ into $V, \mathfrak{I}(A)=1$ for all $A \in \Gamma$ and $\mathfrak{I}(B)<1$ for all $B \in \Delta$. $\mathfrak{I}_{h}$ is an interpretation into $W$. $\mathfrak{I}_{h}(A)=h(\mathfrak{I}(A))=1$ for all $A \in \Gamma$. By strict monotonicity of $h$ and since $\Im(B)<1, h(\Im(B))<1$, and so $\Im_{h}(B)<1$ for all $B \in \Delta$.

Fact 7 (downward Löwenheim-Skolem). For any interpretation $\mathfrak{I}$ (with $|\mathfrak{I}|$ infinite) there is an elementary subinterpretation $\mathfrak{I}^{\prime} \prec \mathfrak{I}$, with countable universe $\left|\mathfrak{I}^{\prime}\right| \subseteq|\mathfrak{I}|$ and $\mathfrak{I}^{\prime}(A)=\mathfrak{I}(A)$ for all $\mathscr{L}^{\mathfrak{I}^{\prime}}$-formulas $A$.

Definition 8. The only sub-formula of an atomic formula $P$ in $\mathscr{L}^{\mathfrak{I}}$ is $P$ itself. The sub-formulas of $A \star B$ for $\star \in\{\rightarrow$ $, \wedge, \vee\}$ are the subformulas of $A$ and of $B$, together with $A \star B$ itself. The sub-formulas of $\forall x A(x)$ and $\exists x A(x)$ with respect to a universe $|\mathfrak{I}|$ are all subformulas of all $A(u)$ for $u \in|\mathfrak{I}|$, together with $\forall x A(x)$ (or, $\exists x A(x)$, respectively) itself.

The set of interpretations of sub-formulas of $A$ under a given interpretation $\mathfrak{I}$ is denoted by

$$
\operatorname{Val}(\mathfrak{I}, A)=\{\mathfrak{I}(B): B \text { sub-formula of } A \text { w.r.t. }|\mathfrak{I}|\} .
$$

\section{The Hypersequent Calculus HGIF}

The method of hypersequents for the axiomatization of non-classical logics was pioneered by Avron [1]. Hypersequent calculi are especially suitable for logics that are characterized semantically by linearly ordered structures, among them Gödel logics. Hypersequent calculi for firstorder Gödel logics can be found in [5, 9]. Ciabattoni extended hypersequent calculi for first-order Gödel logic by rules for $\triangle$ in [10] and studied their proof-theoretic properties.

Definition 9. If $\Gamma$ and $\Delta$ are finite multisets of formulas, and $|\Delta| \leq 1$, then $\Gamma \Rightarrow \Delta$ is an (LJ-) sequent. A finite multiset of sequents is a hypersequent, written $\Gamma_{1} \Rightarrow \Delta_{1}|\ldots| \Gamma_{n} \Rightarrow \Delta_{n}$. Definition 10. The hypersequent calculus HGIF [10] is defined as follows:

Axioms: $A \Rightarrow A$ and $\perp \Rightarrow$. Internal structural rules:

$$
\frac{G \mid \Gamma \Rightarrow \Delta}{G \mid A, \Gamma \Rightarrow \Delta} i w \Rightarrow \quad \frac{G \mid \Gamma \Rightarrow}{G \mid \Gamma \Rightarrow A} \Rightarrow i w \quad \frac{G \mid A, A, \Gamma \Rightarrow \Delta}{G \mid A, \Gamma \Rightarrow \Delta} i c \Rightarrow
$$

External structural rules:

$$
\frac{G}{G \mid \Gamma \Rightarrow \Delta} \text { ew } \quad \frac{G|\Gamma \Rightarrow \Delta| \Gamma \Rightarrow \Delta}{G \mid \Gamma \Rightarrow \Delta} e c
$$

Logical rules:

$$
\begin{aligned}
& \frac{G \mid \Gamma \Rightarrow A}{G \mid \neg A, \Gamma \Rightarrow} \neg \Rightarrow \quad \frac{G \mid A, \Gamma \Rightarrow}{G \mid \Gamma \Rightarrow \neg A} \Rightarrow \neg \\
& \frac{G|A, \Gamma \Rightarrow \Delta \quad G| B, \Gamma \Rightarrow \Delta}{G \mid A \vee B, \Gamma \Rightarrow \Delta} \vee \Rightarrow \frac{G|\Gamma \Rightarrow A \quad G| \Gamma \Rightarrow B}{G \mid \Gamma \Rightarrow A \wedge B} \Rightarrow \wedge \\
& \frac{G \mid \Gamma \Rightarrow A}{G \mid \Gamma \Rightarrow A \vee B} \Rightarrow \vee_{1} \quad \frac{G \mid A, \Gamma \Rightarrow \Delta}{G \mid A \wedge B, \Gamma \Rightarrow \Delta} \wedge \Rightarrow_{1} \\
& \frac{G \mid \Gamma \Rightarrow B}{G \mid \Gamma \Rightarrow A \vee B} \Rightarrow \vee_{2} \quad \frac{G \mid B, \Gamma \Rightarrow \Delta}{G \mid A \wedge B, \Gamma \Rightarrow \Delta} \wedge \Rightarrow_{2} \\
& \frac{G\left|\Gamma_{1} \Rightarrow A \quad G\right| B, \Gamma_{2} \Rightarrow \Delta}{G \mid A \rightarrow B, \Gamma_{1}, \Gamma_{2} \Rightarrow \Delta} \rightarrow \Rightarrow \quad \frac{G \mid A, \Gamma \Rightarrow B}{G \mid \Gamma \Rightarrow A \rightarrow B} \Rightarrow \rightarrow \\
& \frac{G \mid A(t), \Gamma \Rightarrow \Delta}{G \mid(\forall x) A(x), \Gamma \Rightarrow \Delta} \forall \Rightarrow \quad \frac{G \mid \Gamma \Rightarrow A(a)}{G \mid \Gamma \Rightarrow(\forall x) A(x)} \Rightarrow \forall \\
& \frac{G \mid A(a), \Gamma \Rightarrow \Delta}{G \mid(\exists x) A(x), \Gamma \Rightarrow \Delta} \exists \Rightarrow \quad \frac{G \mid \Gamma \Rightarrow A(t)}{G \mid \Gamma \Rightarrow(\exists x) A(x)} \Rightarrow \exists
\end{aligned}
$$

Rules for $\triangle$ :

$$
\begin{array}{ll}
\frac{G \mid A, \Gamma \Rightarrow \Delta}{G \mid \triangle A, \Gamma \Rightarrow \Delta} \triangle \Rightarrow & \frac{G \mid \triangle \Gamma \Rightarrow A}{G \mid \triangle \Gamma \Rightarrow \triangle A} \Rightarrow \triangle \\
\frac{G \mid \triangle \Gamma, \Gamma^{\prime} \Rightarrow \Delta}{G|\triangle \Gamma \Rightarrow| \Gamma^{\prime} \Rightarrow \Delta} \triangle c l &
\end{array}
$$


Cut:

$$
\frac{G|\Gamma \Rightarrow A \quad G| A, \Pi \Rightarrow \Lambda}{G \mid \Gamma, \Pi \Rightarrow \Lambda} c u t
$$

Communication:

$$
\frac{G\left|\Gamma_{1}, \Gamma_{2} \Rightarrow \Delta \quad G\right| \Gamma_{1}, \Gamma_{2} \Rightarrow \Delta^{\prime}}{G\left|\Gamma_{1} \Rightarrow \Delta\right| \Gamma_{2} \Rightarrow \Delta^{\prime}} \mathrm{cm}
$$

The rules $(\Rightarrow \forall)$ and $(\exists \Rightarrow)$ are subject to eigenvariable conditions: the free variable $a$ must not occur in the lower hypersequent.

Definition 11. If $\mathfrak{I}$ is an interpretation into $V$ and $\Gamma \Rightarrow \Delta$ a sequent, define $\mathfrak{I} \models \Gamma \Rightarrow \Delta$ iff $\mathfrak{I}(A)<1$ for all $A \in \Gamma$, or $\Im(B)=1$ for at least one $B \in \Delta$. If $H$ is a hypersequent, define $\mathfrak{I} \models H$ if $\mathfrak{I} \models \Gamma \Rightarrow \Delta$ for at least one $\Gamma \Rightarrow \Delta \in H$.

A hypersequent $H$ is valid in $V$ if $\mathfrak{I}=H$ for all $\mathfrak{I}$ into $V$. Proposition 12. Let $H=\left\langle\Gamma_{i} \Rightarrow \Delta_{i}\right\rangle$ be a hypersequent, and let $\Gamma=\bigcup \Gamma_{i}, \Delta=\bigcup \Delta_{i}$. Then $H$ is valid in $V$ if $\Gamma \vdash_{V} \Delta$.

Theorem 13 (Soundness of HGIF). If $G$ is provable in HGIF, then $\mathfrak{I}=G$ for all $\mathfrak{I}$.

Proof. We shows that for every provable $G$ we have: (*) for every $\mathfrak{I}$, there is a $\Gamma \Rightarrow B \in G$ is so that $\min \{\mathfrak{I}(C): C \in$ $\Gamma\} \leq \mathfrak{I}(B)$. If $(*)$, then also $\mathfrak{I} \models G$. Axioms obviously satisfy the property. Otherwise, $G$ results from a hypersequent $G^{\prime}$ by one of the rules of inference. We give only some cases: $(\Rightarrow \rightarrow)$ If $\min \{\mathfrak{I}(A), \mathfrak{I}(C): C \in \Gamma\} \leq \mathfrak{I}(B)$, then either $\mathfrak{I}(A) \leq \mathfrak{I}(B)$, in which case $\mathfrak{I}(A \rightarrow B)=1$, or $\min \{\Im(C): C \in \Gamma\} \leq \Im(B) \leq \Im(A \rightarrow B) .(\triangle \Rightarrow)$ Obvious, since $\mathfrak{I}(\triangle A) \leq \mathfrak{I}(A) . \quad(\Rightarrow \triangle)$ If $\min \{\mathfrak{I}(\triangle C): C \in \Gamma\} \leq$ $\Im(A)$, then either $\mathfrak{I}(\triangle C)=0$ for some $C$, or $\Im(A)=1$, in which case $\Im(\triangle A)=1$ as well.

Definition 14. We write $\Gamma \vdash \Delta$ if there are $B_{1}, \ldots, B_{n} \in \Delta$ and some finite subset $\Gamma_{0}$ of $\Gamma\left(\Gamma_{0} \subseteq_{f} \Gamma\right)$ so that $\triangle \Gamma_{0} \Rightarrow B_{1} \mid$ $\ldots \mid \triangle \Gamma_{0} \Rightarrow B_{n}$ is provable in HGIF.

Corollary 15. If $\Gamma \vdash \Delta$, then $\Gamma \vdash_{V} \Delta$.

Proof. Follows from Definition 14, Proposition 12 and Theorem 13, together with the fact that if $\mathfrak{I}=\Delta \Gamma \Rightarrow \Delta$, then $\mathfrak{I}=\Gamma \Rightarrow \Delta$.

Completeness proofs for $\mathbf{G}_{V}^{\triangle}$ have usually been given for Hilbert-style systems, which consist of intuitionistic predicate logic extended by additional axioms, such as

$$
\begin{aligned}
\text { QS } & \forall x(C \vee A(x)) \rightarrow(C \vee \forall x A(x)) \\
& (x \text { not free in } C) \\
\text { LIN } & (A \rightarrow B) \vee(B \rightarrow A) \\
\text { ISO }_{0} & \forall x \neg \neg A(x) \rightarrow \neg \neg \forall x A(x) \\
\text { ISO }_{1} & \forall x \neg \triangle A(x) \rightarrow \neg \triangle \exists A(x) \\
\text { FIN }(n) & \left(T \rightarrow p_{1}\right) \vee\left(p_{1} \rightarrow p_{2}\right) \vee \ldots \vee\left(p_{n-2} \rightarrow p_{n-1}\right) \vee \\
& \vee\left(p_{n-1} \rightarrow \perp\right)
\end{aligned}
$$

LIN, of course, is the most fundamental additional schema in this context. Dummett [11] showed that LIN suffices for the axiomatization of propositional infinite-valued Gödel logic. Horn [13] showed that IPL + LIN + QS is complete for first-order intuitionistic logic on linearly-ordered Heyting algebras, which is easily seen to coincide with $\mathbf{G}_{[0,1]}$ (without $\triangle$ ). The authors show elsewhere $[14,7]$ that this system axiomatizes not only $\mathbf{G}_{[0,1]}$ but any $\mathbf{G}_{V}$ where $V$ is an uncountable Gödel set in which 0 is not isolated, and that the addition of $\mathrm{ISO}_{0}$ results in an axiomatization of $\mathbf{G}_{V}$ for any uncountable Gödel set in which 0 is isolated.

Takeuti and Titani [18] have used and axiomatized the $\triangle$ operator (there denoted by $\square$ ) in the context of their intuitionistic fuzzy logic, which coincides with $\mathbf{G}_{[0,1]}^{\triangle}$. The $\triangle$ operator for $[0,1]$-valued Gödel logics was also introduced in [2], and was given an axiomatization there using the following axioms:

$$
\begin{array}{ll}
\triangle 1 & \triangle A \vee \neg \triangle A \\
\triangle 2 & \triangle(A \vee B) \rightarrow(\triangle A \vee \triangle B) \\
\triangle 3 & \triangle A \rightarrow A \\
\triangle 4 & \triangle A \rightarrow \triangle \triangle A \\
\triangle 5 & \triangle(A \rightarrow B) \rightarrow(\triangle A \rightarrow \triangle B) \\
\triangle R & A \vdash \Delta A
\end{array}
$$

The hypersequent axiomatization for $\triangle$ above was introduced and shown complete for the propositional case in [5].

It is an easy exercise to show that the various axioms listed above (with the exception of $\mathrm{FIN}(n), \mathrm{ISO}_{0}, \mathrm{ISO}_{1}$ ) are indeed derivable in HGIF. Conversely, using the translation of hypersequents $G=\left\langle\Gamma_{i} \Rightarrow \Delta_{i}\right\rangle_{i}$ into the formulas $G^{*}=\bigvee_{i}\left(\wedge \Gamma_{i} \rightarrow B_{i}\right)$, where $B_{i} \equiv \perp$ if $\Delta_{i}=\emptyset$, and $=B_{i}$ if $\Delta_{i}=\left\{B_{i}\right\}$, one can show that $G$ is provable in HGIF iff $G^{*}$ is provable in the corresponding Hilbert-type system. This latter fact has been used in completeness arguments for HIF [3] and related systems hitherto.

\section{Topology of Gödel Sets}

All the following notations, lemmas, theorems are carried out within the framework of Polish spaces, which are separable, completely metrizable topological spaces. For our discussion it is only necessary to know that $\mathbb{R}$ is such a Polish space.

Definition 16 (limit point, perfect space, perfect set). A limit point of a topological space is a point that is not isolated, i.e. for every open neighborhood $U$ of $x$ there is a point $y \in U$ with $y \neq x$. A space is perfect if all its points are limit points. A set $P \subseteq \mathbb{R}$ is perfect if it is closed and together with the topology induced from $\mathbb{R}$ is a perfect space.

It is obvious that all (non-trivial) closed intervals are perfect sets, also all countable unions of (non-trivial) intervals. But all these sets generated from closed intervals have the property that they are 'everywhere dense', i.e., contained in the closure of their inner component. There is an example 
of a set which is perfect but is nowhere dense, the Cantor set:

Example (Cantor Set). The set of all numbers in the unit interval which can be expressed in triadic notation only by digits 0 and 2 is called Cantor set $\mathbb{D}$.

Fact 17. The Cantor set is perfect.

It is possible to embed the Cauchy space into any perfect space, yielding the following proposition:

Proposition 18. If $X$ is a nonempty perfect Polish space, then the cardinality of $X$ is $2^{\aleph_{0}}$ and therefore, all nonempty perfect subsets, too, have cardinality of the continuum.

Every Polish space can be partitioned into a perfect kernel and a countable rest. This is the well known CantorBendixon Theorem:

Theorem 19 (Cantor-Bendixon). Let $X$ be a Polish space. Then $X$ can be uniquely written as $X=P \cup C$, with $P$ a perfect subset of $X$ and $C$ countable and open. The subset $P$ is called the perfect kernel of $X$ (denoted with $X^{\infty}$ ).

As a corollary we obtain that any uncountable Polish space contains a perfect set, and therefore, has cardinality $2^{\aleph_{0}}$.

Lemma 20. Suppose that $M \subseteq[0,1]$ is countable and $P \subseteq$ $[0,1]$ is perfect. Then there is a strictly monotone continuous map $h: M \rightarrow P$. Furthermore, if both $M$ and $P$ contain 0 or 1 , then hreserves 0 and 1 .

Proof. Let $w$ be an injective monotone continuous map from $M$ into $2^{\omega}$, i.e. $w(m)$ is a fixed binary representation of $m$. For dyadic rational numbers (i.e. those with different binary representations) we fix one possible.

Let $i$ be the natural bijection from $2^{\omega}$ (the set of infinite $\{0,1\}$-sequences, ordered lexicographically) onto $\mathbb{D}$, the Cantor set. $i$ is an order preserving homeomorphism.

Since $P$ is perfect, we can find a continuous strictly monotone map $c$ from the Cantor set $\mathbb{D} \subseteq[0,1]$ into $P$, and if $P \ni 0,1, c$ can be chosen so that $c(0)=0, c(1)=1$.

Now $h=c \circ i \circ w$ is also a strictly monotone continuous map from $M$ into $P$, and $h(0)=0$, if $0 \in M$, and $h(1)=1$, if $1 \in M$.

Corollary 21. A Gödel set is uncountable iff it contains a non-trivial dense linear subordering.

Proof. If: Every countable non-trivial dense linear order has order type $\eta, \mathbf{1}+\eta, \eta+\mathbf{1}$, or $\mathbf{1}+\eta+\mathbf{1}[15$, Corollary 2.9], where $\eta$ is the order type of $\mathbb{Q}$. The completion of any ordering of order type $\eta$ has order type $\lambda$, the order type of $\mathbb{R}[15$, Theorem 2.30$]$, thus the truth value set must be uncountable.

Only if: By Theorem $19, V^{\infty}$ is nonempty. Take $M=\mathbb{Q}$ in Lemma 20, and $P=V^{\infty}$. The image of $M$ under the $\mathbf{G}$ embedding from $M$ into the perfect kernel of $V$ is a nontrivial dense linear subordering.
Theorem 22. Let $V$ be a Gödel set with non-empty perfect kernel $V^{\infty}$, and 0 and $1 \in V^{\infty}$. Then $\Gamma \vdash_{V} B$ iff $\Gamma \vdash_{[0,1]} B$.

Proof. If: Lemma 6.

Only if: Suppose $\Gamma \nVdash_{[0,1]} B$, i.e., for some $\mathfrak{I}$ into $[0,1]$, $\mathfrak{I}(A)=1$ for all $A \in \Gamma$ and $\mathfrak{I}(B)<1$. By Fact 7 , there is an $\mathfrak{I}^{\prime} \prec \mathfrak{I}$ such that $|\mathfrak{I}|$ is countable. Then $M=\bigcup\left\{\operatorname{Val}\left(\mathfrak{I}^{\prime}, A\right)\right.$ : $A \in \Gamma \cup\{B\}\}$ has cardinality at most $\aleph_{0}$, thus there exists a $b \in(0,1)$ such that $b \notin M, \mathfrak{I}^{\prime}(B)<b<1$. Furthermore, there are values $\ell$ and $u, \ell<u$, and such that $[0, \ell] \cap V$ and $[u, 1] \cap V$ are perfect. By Lemma 20, there are continuous strictly monotone $h_{\ell}:[0, b] \cap(M \cup\{b\}) \rightarrow[0, \ell] \cap V$ with $h_{\ell}(0)=0$, and $h_{u}:[b, 1] \cap(M \cup\{b\}) \rightarrow[u, 1] \cap V$ with $h_{u}(1)=1$. Define $\mathfrak{J}$ into $V$ by

$$
P^{\mathfrak{J}}(\vec{u})= \begin{cases}h_{\ell}\left(P^{\Im^{\prime}}(\vec{u})\right) & \text { if } 0 \leq P^{\Im^{\prime}}(\vec{u})<b \\ h_{u}\left(P^{\Im^{\prime}}(\vec{u})\right) & \text { if } b<P^{\Im^{\prime}}(\vec{u}) \leq 1\end{cases}
$$

for all atomic $A$. By induction one shows that the above property extends to all formulas. Since $\mathfrak{I}^{\prime}(A)=1$ for all $A \in \Gamma$ and $\mathfrak{I}^{\prime}(B)<b$, we have that $\mathfrak{J}(A)=1$ for all $A \in \Gamma$, and $\mathfrak{J}(B)<\ell<1$, and thus $\Gamma \nVdash_{V} B$.

\section{Completeness of HGIF}

The main result of this paper is a direct proof of strong completeness for HGIF for any Gödel set $V$ which is uncountable and 0,1 contained in the perfect kernel of $V$. Due to Theorem 22 we only have to show completeness for $V=[0,1]$. We use the method of Takano [16].

Theorem 23. If $\Gamma \vdash_{[0,1]} A$, then $\Gamma \vdash A$.

The proof proceeds in several steps. We show that if $\Gamma \nvdash A$, then there is an interpretation $\mathfrak{I}$ into $[0,1]$ so that $\mathfrak{I}(\Gamma)=1$ but $\mathfrak{I}(A)<1$.

Lemma 24. Suppose $\Gamma \nvdash A$. Let $a_{1}, a_{2}, \ldots$ be a sequence of free variables which do not occur in $\Gamma \cup\{A\}$, let $\mathcal{T}$ be the set of all terms in the language of $\Gamma \cup\{A\}$ together with the new variables $a_{1}, a_{2}, \ldots$, and let $\mathcal{F}=\left\{F_{1}, F_{2}, \ldots\right\}$ be an enumeration of the formulas in this language in which $a_{i}$ does not appear in $F_{1}, \ldots, F_{i}$ and in which each formula appears infinitely often.

Let $\Gamma_{0}=\Gamma$ and $\Delta_{0}=\{A\}$. (a) If $\Gamma_{n} \vdash \Delta_{n} \cup\left\{F_{n}\right\}$, then $\Gamma_{n+1}=\Gamma_{n} \cup\left\{F_{n}\right\}$ and $\Delta_{n+1}=\Delta_{n}$. (b) If $\Gamma_{n} \nvdash \Delta_{n} \cup\left\{F_{n}\right\}$, then $\Gamma_{n+1}=\Gamma_{n}$ and $\Delta_{n+1}=\Delta_{n} \cup\left\{F_{n}, B\left(a_{n}\right)\right\}$ if $F_{n} \equiv \forall x B(x)$, and $\Delta_{n+1}=\Delta_{n} \cup\left\{F_{n}\right\}$ otherwise.

Then $\Gamma_{n} \nvdash \Delta_{n}$ for all $n$.

Proof. By the assumption of the lemma, $\Gamma_{0} \nvdash \Delta_{0}$.

Suppose that $\Gamma_{n} \nvdash \Delta_{n}$, we show that this is also the case for $n+1$.

(a) Suppose that $\Gamma_{n} \cup\left\{F_{n}\right\} \vdash \Delta_{n}$. Then for some $\Gamma^{\prime} \subseteq_{f} \Gamma_{n}$ and $B_{1}, \ldots, B_{k} \in \Delta_{n}, \triangle \Gamma^{\prime}, \triangle F_{n} \Rightarrow B_{1}|\ldots| \triangle \Gamma^{\prime}, \triangle F_{n} \Rightarrow B_{k}$ is provable. But by the assumption of case (a), also $\triangle \Gamma^{\prime} \Rightarrow B_{1}|\ldots| \triangle \Gamma^{\prime} \Rightarrow B_{k} \mid \triangle \Gamma^{\prime} \Rightarrow F_{n}$ is provable. From 
the latter we get $\triangle \Gamma^{\prime} \Rightarrow B_{1}|\ldots| \triangle \Gamma^{\prime} \Rightarrow B_{k} \mid \triangle \Gamma^{\prime} \Rightarrow \triangle F_{n}$ by $(\triangle \mathrm{cl})$, and then $k$ applications of cut result in $\triangle \Gamma^{\prime} \Rightarrow B_{1}$ $\ldots \mid \triangle \Gamma^{\prime} \Rightarrow B_{k}$. But by induction hypothesis, $\Gamma_{n} \nvdash \Delta_{n}$.

(b) In this case, it is obvious that $\Gamma_{n+1} \nvdash \Delta_{n+1}$ if $F_{n} \not \equiv \forall x B(x)$. Now suppose $F_{n} \equiv \forall x B(x)$, and $\Gamma_{n} \vdash \Delta_{n} \cup$ $\left\{F_{n}, B\left(a_{n}\right)\right\}$. Then for some $\Gamma^{\prime} \subseteq_{f} \Gamma_{n}$ and $B_{1}, \ldots, B_{k} \in \Delta_{n}$, HGIF proves $\triangle \Gamma^{\prime} \Rightarrow B_{1}|\ldots| \triangle \Gamma^{\prime} \Rightarrow B_{k}\left|\triangle \Gamma^{\prime} \Rightarrow \forall x B(x)\right|$ $\triangle \Gamma^{\prime} \Rightarrow B\left(a_{n}\right)$. Since $a_{n}$ does not appear in $F_{n}$ or $\Gamma_{n}, \Delta_{n}, a_{n}$ satisfies the eigenvariable condition. By $(\Rightarrow \forall)$ and external contraction, we'd have $\Gamma_{n} \vdash \Delta_{n} \cup\left\{F_{n}\right\}$ contrary to the assumption.

Let $\Gamma^{*}=\bigcup_{i=0}^{\infty} \Gamma_{i}$ and $\Delta^{*}=\bigcup_{i=0}^{\infty} \Delta_{i}$ as defined in the preceding lemma. We have:

Lemma 25. (1) $\Gamma^{*} \nvdash \Delta^{*}$. (2) $\Gamma^{*}=\mathcal{F} \backslash \Delta^{*}$. (3) If $\Gamma^{*} \vdash$ $\left\{B_{1}, \ldots, B_{n}\right\}$, then $B_{i} \in \Gamma^{*}$ for some $i$. In particular, if $\Gamma^{*} \vdash B$, then $B \in \Gamma^{*}$. (4) If $B(t) \in \Gamma^{*}$ for every $t \in \mathcal{T}$, then $\forall x B(x) \in \Gamma^{*}$.

Proof. (1) Otherwise there would be a $k$ so that $\Gamma_{k} \vdash \Delta_{k}$, contrary to Lemma 24. (2) Each $F_{n}$ is either in $\Gamma_{n+1}$ or $\Delta_{n+1}$, and if for some $n, F_{n} \in \Gamma^{*} \cap \Delta^{*}$, there would be a $k$ so that $F_{n} \in \Gamma_{k} \cap \Delta_{k}$, which is impossible since $\Gamma_{k} \nvdash \Delta_{k}$. (3) Suppose not, then for $i=1, \ldots, n, B_{i} \notin \Gamma^{*}$, and hence, by (2), $B_{i} \in \Delta^{*}$. But then $\Gamma^{*} \vdash \Delta^{*}$, contradicting (1). (4) Otherwise, by (2), $\forall x B(x) \in \Delta^{*}$ and so there is some $n$ so that $\forall x B(x)=F_{n}$ and $\Delta_{n+1}$ contains $\forall x B(x)$ and $B\left(a_{n}\right)$. But, again by (2), then $B\left(a_{n}\right) \notin \Gamma^{*}$.

We will make use of (3) often in what follows, in particular the case where $i=1$ (i.e., $\Gamma^{*}$ is closed under provability). Note in particular that if $\Gamma^{*} \Rightarrow B$ is provable, then $\triangle \Gamma^{*} \Rightarrow B$ is also provable by $(\triangle \Rightarrow)$, and hence $\Gamma^{*} \vdash B$.

Define relations $\preceq$ and $\equiv$ on $\mathcal{F}$ by

$$
B \preceq C \Leftrightarrow B \rightarrow C \in \Gamma^{*} \quad \text { and } \quad B \equiv C \Leftrightarrow B \preceq C \wedge C \preceq B .
$$

Then $\preceq$ is reflexive and transitive, since for every $B, \vdash B \rightarrow$ $B$ and so $B \rightarrow B \in \Gamma^{*}$, and if $B \rightarrow C \in \Gamma^{*}$ and $C \rightarrow D \in \Gamma^{*}$ then $B \rightarrow D \in \Gamma^{*}$, since $B \rightarrow C, C \rightarrow D \vdash B \rightarrow D$. Hence, $\equiv$ is an equivalence relation on $\mathcal{F}$. For every $B$ in $\mathcal{F}$ we let $|B|$ be the equivalence class under $\equiv$ to which $B$ belongs, and $\mathcal{F} / \equiv$ the set of all equivalence classes. Next we define the relation $\leq$ on $\mathcal{F} / \equiv$ by

$$
|B| \leq|C| \Leftrightarrow B \preceq C \Leftrightarrow B \rightarrow C \in \Gamma^{*} .
$$

Obviously, $\leq$ is independent of the choice of representatives $B, C$.

Lemma 26. $\langle\mathcal{F} / \equiv, \leq\rangle$ is a countably linearly ordered structure with distinct maximal element $|\top|$ and minimal element $|\perp|$.
Proof. Since $\mathcal{F}$ is countably infinite, $\mathcal{F} / \equiv$ is countable. For every $B$ and $C, \vdash\{B \rightarrow C, C \rightarrow B\}$ by

$$
\begin{gathered}
\frac{B \Rightarrow B \mid C \Rightarrow C}{B, C \Rightarrow B \mid B, C \Rightarrow C}= \\
\frac{B \Rightarrow C \mid C \Rightarrow B}{\Rightarrow B \rightarrow C \mid \Rightarrow C \rightarrow B} \Rightarrow \rightarrow
\end{gathered}
$$

and so either $B \rightarrow C \in \Gamma^{*}$ or $C \rightarrow B \in \Gamma^{*}$ by (3), hence $\leq$ is linear. For every $B, \vdash B \rightarrow \top$ and $\vdash \perp \rightarrow B$, and so $B \rightarrow \top \in \Gamma^{*}$ and $\perp \rightarrow B \in \Gamma^{*}$, hence $|\top|$ and $|\perp|$ are the maximal and minimal elements, respectively. Pick any $A$ in $\Delta^{*}$. Since $\top \rightarrow \perp \vdash A$, and $A \notin \Gamma^{*}, \top \rightarrow \perp \notin \Gamma^{*}$, so $|\top| \neq|\perp|$.

We abbreviate $|T|$ by $\mathbf{1}$ and $|\perp|$ by $\mathbf{0}$.

Lemma 27. The following properties hold in $\langle\mathcal{F} \mid \equiv, \leq\rangle$ :

1. $|B|=\mathbf{1} \Leftrightarrow B \in \Gamma^{*}$.

2. $|B \wedge C|=\min \{|B|,|C|\}$.

3. $|B \vee C|=\max \{|B|,|C|\}$.

4. $|B \rightarrow C|=1$ if $|B| \leq|C|,|B \rightarrow C|=|C|$ otherwise.

5. $|\neg B|=\mathbf{1}$ if $|B|=\mathbf{0} ;|\neg B|=\mathbf{0}$ otherwise.

6. $|\triangle B|=\mathbf{1}$ if $|B|=\mathbf{1} ;|\triangle B|=\mathbf{0}$ otherwise.

7. $|\exists x B(x)|=\sup \{|B(t)|: t \in \mathcal{T}\}$.

8. $|\forall x B(x)|=\inf \{|B(t)|: t \in \mathcal{T}\}$.

Proof. (1) If $|B|=\mathbf{1}$, then $\top \rightarrow B \in \Gamma^{*}$, and hence $B \in \Gamma^{*}$. And if $B \in \Gamma^{*}$, then $\top \rightarrow B \in \Gamma^{*}$ since $B \vdash \top \rightarrow B$. So $|\top| \leq|B|$. It follows that $|\top|=|B|$ as also $|B| \leq|\top|$.

(2) From $\vdash B \wedge C \rightarrow B, \vdash B \wedge C \rightarrow C$ and $D \rightarrow B, D \rightarrow C \vdash$ $D \rightarrow B \wedge C$ for every $D$, it follows that $|B \wedge C|=\inf \{|B|,|C|\}$, from which (2) follows since $\leq$ is linear. (3) is proved analogously.

(4) If $|B| \leq|C|$, then $B \rightarrow C \in \Gamma^{*}$, and since $\top \in \Gamma^{*}$ as well, $|B \rightarrow C|=\mathbf{1}$. Now suppose that $|B| \not \leq|C|$. From $\vdash$ $B \wedge(B \rightarrow C) \rightarrow C$ it follows that $\min \{|B|,|B \rightarrow C|\} \leq|C|$. Because $|B| \not \leq|C|, \min \{|B|,|B \rightarrow C|\} \neq|B|$, hence $\mid B \rightarrow$ $C|\leq| C \mid$. On the other hand, $\vdash C \rightarrow(B \rightarrow C)$, so $|C| \leq \mid B \rightarrow$ $C \mid$.

(5) Immediate by (4).

(6) Suppose $|B|=\mathbf{1}$, i.e., $B \in \Gamma^{*}$ and hence $\Gamma^{*} \vdash B$, i.e., for some $\Gamma^{\prime} \subseteq_{f} \Gamma^{*}, \triangle \Gamma^{\prime} \Rightarrow B$. Then, by $(\Rightarrow \triangle), \triangle \Gamma^{*} \Rightarrow \triangle B$ is also provable, hence $\Gamma^{*} \vdash \triangle B$, hence $\triangle B \in \Gamma^{*}$.

Now suppose $|B| \neq \mathbf{1}$, i.e., $B \notin \Gamma^{*}$. Then $\triangle B \notin \Gamma^{*}$, since $\vdash \triangle B \rightarrow B$. Using the derivation

$$
\begin{gathered}
\frac{\triangle B \Rightarrow \triangle B}{\triangle B \Rightarrow \mid \Rightarrow \triangle B} \Delta c l \\
\Rightarrow \neg \triangle B \mid \Rightarrow \triangle B
\end{gathered}
$$

one sees that either $\triangle B \in \Gamma^{*}$ or $\neg \triangle B \in \Gamma^{*}$. Since $\triangle B \notin \Gamma^{*}$, $\neg \triangle B \in \Gamma^{*}$, i.e., $|\triangle B|=\mathbf{0}$. 
(7) Since $\vdash B(t) \rightarrow \exists x B(x),|B(t)| \leq|\exists x B(x)|$ for every $t \in \mathcal{T}$. On the other hand, for every $D$ without $x$ free,

$$
\begin{aligned}
& |B(t)| \leq|D| \\
\Leftrightarrow & B(t) \rightarrow D \in \Gamma^{*} \\
\Rightarrow & \forall x(B(x) \rightarrow D) \in \Gamma^{*} \\
\Rightarrow & \exists x B(x) \rightarrow D \in \Gamma^{*} \\
\Leftrightarrow & |\exists x B(x)| \leq|D| .
\end{aligned}
$$

\section{for every $t \in \mathcal{T}$}

for every $t \in \mathcal{T}$

by property (5) of $\Gamma^{*}$ since $\forall x(B(x) \rightarrow D) \vdash \exists x B(x) \rightarrow D$

(8) is proved analogously.

Proof of Completeness Theorem. Suppose $\Gamma \nvdash A$. Then, by the preceding lemmas, $\mathfrak{J}$ defined by $|\mathfrak{J}|=\mathcal{T}$ and $P^{\mathfrak{J}}(\vec{u})=$ $|P(\vec{u})|$ is an interpretation into $\langle\mathcal{F} / \equiv, \leq\rangle$ with $\mathfrak{J}(A)<\mathbf{1}$ and $\mathfrak{J}(B)=\mathbf{1}$ for all $B \in \Gamma .\langle\mathcal{F} / \equiv, \leq\rangle$ is countable, let $\mathbf{0}=a_{0}$, $\mathbf{1}=a_{1}, a_{2}, \ldots$ be an enumeration. Define $h(\mathbf{0})=0, h(\mathbf{1})=$ 1 , and for $n>1$, let $h\left(a_{n}\right)=\left(h\left(a_{\ell}\right)+h\left(a_{u}\right)\right) / 2$ where $a_{\ell}=$ $\max \left\{a_{i}: i<n, a_{i}<a_{n}\right\}$, and $a_{u}=\min \left\{a_{i}: i<n, a_{i}>a_{n}\right\}$. Then $h:\langle\mathcal{F} / \equiv, \leq\rangle \rightarrow[0,1] \cap \mathbb{Q}$ is clearly strictly monotone and preserves infs and sups.

By Lemma 20 there exists a G-embedding $h^{\prime}:[0,1] \cap$ $\mathbb{Q} \rightarrow[0,1]$. Then $\mathfrak{I}=\mathfrak{J}_{h^{\prime} \circ h}$ is an interpretation into $[0,1]$ with $\Im(A)<1$ and $\Im(B)=1$ for all $B \in \Gamma$.

Theorem 28. Suppose $0,1 \in V^{\infty}$. Then $\Gamma \vdash A$ iff $\Gamma \vdash_{V} A$.

Proof. By Theorems 22, 13 and 23.

\section{Conclusion and Open Problems}

The contributions of the present paper are mainly the direct method of the completeness proof used for HGIF. It combines ideas of Takano's with the work of Baaz, Ciabattoni, and others on hypersequent formulations of first-order infinite valued logics. The main remaining open problem is the formulation of hypersequent rules corresponding to $\mathrm{ISO}_{0}$ and $\mathrm{ISO}_{1}$, which would result in an axiomatization of $\mathbf{G}_{V}^{\triangle}$ for $V$ uncountable and with 0 or 1 isolated. The difficulty in this respect is that the rules must be formulated so that cut-elimination holds for HIF [3] and HGIF [10] can also be adapted.

\section{References}

[1] A. Avron. Hypersequents, logical consequence and intermediate logics for concurrency. Ann. Math. Artificial Intelligence, 4:225-248, 1991.

[2] M. Baaz. Infinite-valued Gödel logics with 0-1-projections and relativizations. In P. Hájek, ed., Proc. Gödel'96, Logic Foundations of Mathematics, Computer Science and Physics - Kurt Gödel's Legacy, Lecture Notes in Logic 6, 23-33. Springer, 1996.
[3] M. Baaz and A. Ciabattoni. A Schütte-Tait style cutelimination proof for first-order Gödel logic. In Automated Reasoning with Analytic Tableaux and Related Methods, tableaux 2002. Proceedings, LNAI 2381, 24-38. Springer, 2002.

[4] M. Baaz, A. Ciabattoni, and R. Zach. Quantified propositional Gödel logic. In A. Voronkov and M. Parigot, editors, Logic for Programming and Automated Reasoning. 7th International Conference, LPAR 2000, 240-256, Berlin, 2000. Springer. LNAI 1955.

[5] M. Baaz, C. G. Fermüller, and A. Ciabattoni. Hypersequent calculi for Gödel logics-a survey. J. Logic and Computation, 13:835-861, 2003.

[6] M. Baaz, N. Preining, and R. Zach. Characterization of the axiomatizable prenex fragments of first-order Gödel logics. In 33rd International Symposium on Multiple-valued Logic. May 2003, Tokyo, Japan. Proceedings, 175-180. IEEE Press, Los Alamitos, 2003.

[7] M. Baaz, N. Preining, and R. Zach. First-order Gödel logics. submitted, 2006.

[8] M. Baaz and H. Veith. An axiomatization of quantified propositional Gödel logic using the Takeuti-Titani rule. In S. Buss, P. Hájek, and P. Pudlák, editors, Proceedings of the Logic Colloquium '98, Prague, LNL 13, 74-87. ASL, 2000.

[9] M. Baaz and R. Zach. Hypersequents and the proof theory of intuitionistic fuzzy logic. In P. G. Clote and H. Schwichtenberg, editors, Computer Science Logic CSL'2000. Proceedings, LNCS 1862, 178-201. Springer, 2000.

[10] A. Ciabattoni. A proof-theoretical investigation of global intuitionistic (fuzzy) logic. Archive Math. Logic, 44:435457, 2005

[11] M. Dummett. A propositional logic with denumerable matrix. J. Symbolic Logic, 24:96-107, 1959.

[12] K. Gödel. Zum Intuitionistischen Aussagenkalkül. Ergebnisse eines mathematischen Kolloquiums, 4:34-38, 1933.

[13] A. Horn. Logic with truth values in a linearly ordered Heyting algebra. J. Symbolic Logic, 34(3):395-409, 1969.

[14] N. Preining. Complete Recursive Axiomatizability of Gödel Logics. PhD thesis, Vienna University of Technology, Austria, 2003.

[15] J. G. Rosenstein. Linear Orderings. Academic Press, 1982.

[16] M. Takano. Another proof of the strong completeness of the intuitionistic fuzzy logic. Tsukuba J. Math., 11(1):101-105, 1987.

[17] G. Takeuti and S. Titani. Intuitionistic fuzzy logic and intuitionistic fuzzy set theory. J. Symbolic Logic, 49:851-866, 1984.

[18] G. Takeuti and S. Titani. Global intuitionistic fuzzy set theory. In The Mathematics of Fuzzy Systems, 291-301. TUVVerlag, 1986. 